\title{
O impacto emocional da cirurgia bariátrica em pacientes com obesidade mórbida
}

\author{
The emotional impact of bariatric surgery in patients with morbid obesity
}

\author{
Cristiano Waihrich Leal ${ }^{1}$, Nelma Baldin ${ }^{2}$ \\ ${ }^{1}$ Mestrando. Professor, Universidade da Região de Joinville (UNIVILLE), Joinville, SC. ${ }^{2}$ Doutora. Professora de pós-graduação, UNIVILLE.
}

\begin{abstract}
Resumo
A cirurgia bariátrica é um método que tem sido utilizado para o emagrecimento e resgate da saúde. Em muitos casos, porém, o emagrecimento súbito incorre em quadros psiquiátricos, tais como: sintomas depressivos, ansiedade, uso de substâncias, alterações comportamentais e ideação suicida, dentre outras situações, associadas às mudanças emocionais que o paciente vivencia com o novo estado físico e psíquico. O atual estudo teve como objetivo analisar as expectativas, fantasias, resultados, dificuldades e frustrações enfrentadas após o procedimento cirúrgico. Realizou-se um relato de caso das informações colhidas com seis pacientes em acompanhamento num hospital público de Santa Catarina e que desenvolveram sintomas de sofrimento psíquico após a cirurgia bariátrica. Cada paciente participou de duas sessões de entrevistas semi-estruturadas. A pesquisa foi submetida e aprovada pela comissão de ética da Universidade de Joinville. Os resultados obtidos demonstram que as mudanças psicológicas decorrentes da cirurgia foram marcantes. Em determinados casos, as entrevistadas expressaram expectativas além do emagrecimento, como a resolução dos conflitos interpessoais e conjugais, assim como mudanças de traços definidos de suas personalidades. Constatou-se, também, o uso de substâncias (álcool e tabaco) associado a comportamentos de risco (envolvimento extraconjugal e direção perigosa). O problema da obesidade ficou evidenciado como parte de uma complexa situação que envolve o estado físico e emocional das pessoas, e sua resolução, por vezes, expõe as dificuldades e as limitações psíquicas dos pacientes.
\end{abstract}

Descritores: Obesidade mórbida, cirurgia bariátrica, adaptação psicológica.

\begin{abstract}
Bariatric surgery has been used for health recovery and weight loss. In many cases, however, abrupt weight loss produces psychiatric symptoms, such as depression, anxiety, use of drugs, behavioral changes and suicidal ideation, among other situations, all related to the emotional changes faced by the patient in his new physical and chemical state. This study aimed at analyzing hopes, fantasies, results, difficulties, and frustrations present after a surgical procedure. This case report resulted from the data collected from six patients being followed at a public hospital in Santa Catarina, Brazil, who developed symptoms of psychic suffering after the bariatric surgery. Each patient participated in two sessions of semi-structured interviews. This research was approved by the ethics committee of Universidade de Joinville. Results show that psychological changes resulting from the bariatric surgery were remarkable. In some cases, patients expressed expectations beyond weight loss, such as resolution of marital and interpersonal conflicts, as well as changes in stable personality traits. Use of substances (alcohol and tobacco) associated with risk behavior (extra-marital relationships and dangerous driving) was also detected. The problem of obesity was evidenced as part of a complex situation that involves both physical and mental status, and its solution sometimes exposes the patient's difficulties and psychological limitations.
\end{abstract}

Keywords: Morbid obesity, bariatric surgery, psychological adaptation.

\section{Correspondência:}

Cristiano Waihrich Leal, Rua Blumenau, 178/307, CEP 89204-250, Joinville, SC. Tel./Fax: (47) 3423.2041. E-mail: cwleal@terra.com.br 


\section{Introdução}

Técnicas modernas de intervenção quanto ao emagrecimento - dentre as quais, no caso, a cirurgia bariátrica - têm sido desenvolvidas para o tratamento da obesidade (como método de emagrecimento), em especial na sua forma mórbida. No entanto, mesmo mostrando-se extremamente eficazes, com resultados extremamente satisfatórios para um público considerável, tais técnicas de emagrecimento também têm se defrontado com um outro tipo de problema às pessoas que as praticam: apresentação de alterações psicológicas após a realização da cirurgia ${ }^{1}$.

Por tratar-se de uma entidade nova, as complicações psiquiátricas após a cirurgia ainda não dispõem de instrumentos para mensurá-las² ${ }^{2}$ Em muitos casos, o emagrecimento súbito incorre em quadros psiquiátricos, tais como: depressão, ansiedade, alcoolismo e gastos excessivos, dentre outras situações.

Pacientes mais jovens e com maior consumo de álcool resultaram em pior evolução pós-cirúrgica ${ }^{3}$, assim como 1/3 dos pacientes podem apresentar piora no relacionamento conjugal ${ }^{4}$.

Convém lembrar que a obesidade muitas vezes está associada a alterações psiquiátricas, podendo atingir prevalência de até $50 \%$ dos pacientes obesos mórbidos ${ }^{3}$, assim como estar ligada a maior dificuldade nos relacionamentos interpessoais ${ }^{5}$. Os transtornos de personalidade indicaram menor perda de peso após a cirurgia $^{6,7}$. Destaca-se que certas alterações psiquiátricas são contra-indicações absolutas para a realização da cirurgia bariátrica, dentre elas: psicose em atividade, uso corrente de álcool ou drogas, situação de vida caótica e inabilidade para cooperar com o tratamento pós-cirúrgico ${ }^{8}$.

Por outro lado, estudos de longo prazo evidenciaram abrandamento em traços do caráter oral, tais como dependência, submissão e insegurança, ao passo que se detectou um aumento em traços e comportamentos obsessivos ${ }^{9}$.

Fatores estéticos, não raro, são os maiores motivadores na busca pela cirurgia bariátrica, reforçados por preconceitos de que pacientes obesos são mais frágeis e estigmatizados socialmente ${ }^{10}$.

$\mathrm{O}$ excesso alimentar pode ser visto associado a estímulos reforçadores na hora de se alimentar e estímulos negativos ou aversivos tardios, que nem sempre são discriminados pelo indivíduo, assim como ligado a fatores herdados e ambientais ${ }^{11,12}$. Por outro lado, também está associado a fatores comportamentais, como produto de um aprendizado ${ }^{13}$. O uso do alimento, não raro, está ligado à fonte de ganhos psíquicos secundários ${ }^{14}$.

Com base nesses pressupostos, este estudo tem como objetivo o relato dos fenômenos psicológicos envolvidos no procedimento da cirurgia bariátrica e suas implicações, bem como as expectativas, as fantasias, os resultados, as dificuldades e as frustrações enfrentadas pelo paciente no estado pós-cirúrgico.

Para tanto, foram selecionadas seis pacientes mulheres - que estavam em acompanhamento num hospital público e que desenvolveram sintomas de sofrimento psíquico após a realização da cirurgia.

\section{Descrição dos casos}

Das seis entrevistadas, cinco delas já se encontravam sob tratamento medicamentoso com antidepressivos antes do contato com os procedimentos da pesquisa.

Considerando-se a situação geral das entrevistadas, verificou-se que, antes da cirurgia, a média de peso das mesmas era de $127,5 \mathrm{~kg}$. Esse quadro tendeu para menos, após a cirurgia, com uma redução média de $39,3 \%$ no peso. No entanto, apesar da perda de peso, as entrevistadas não demonstraram estar satisfeitas.

A entrevistada 1, casada, 26 anos, buscou a cirurgia por motivações estéticas. Após o procedimento, sua vida teve grandes mudanças: teve um envolvimento extraconjugal, por sentir-se "mais desejada pelos homens", assim como passou a ter uma postura mais ativa no casamento. Relata que "antes era ele [o marido] quem aprontava, agora sou eu quem dá o troco”. Devido à obesidade, deixava de realizar várias atividades, até mesmo as mais rotineiras, como ir a uma loja para comprar roupas. Diz que sentia vergonha e que, por esse motivo, pedia para a mãe ou cunhada irem até o local e buscar roupas para que experimentasse em casa. Chega a ser enfática em rechaçar a obesidade: "Eu me odiava!". Relata as mudanças nos papéis do casal com a seguinte afirmativa: "Quando eu era gorda, já foi bastante difícil no meu casamento... Não sei se era por ser gorda ou se era da minha cabeça. Já foi bem complicado... Antes era o meu marido o problemático (sic)... Hoje eu que sou chata, implico com tudo".

Após a cirurgia, desenvolveu o hábito de beber com maior freqüência. Nos últimos meses, apresentou um agravamento de seus sintomas, chegando a ter uma tentativa de suicídio, por acreditar que estava engordando novamente. Afirmou que acreditava ser impossível voltar a engordar.

A entrevistada 2, casada, 36 anos, relatou que o alimento sempre serviu como "fuga" para suas angústias, fato que teve início na sua infância, por ter um relacionamento conflituoso com o pai. Conta que, naquele período, costumava acordar no meio da noite e comer escondida para evitar represálias do pai. Refere que a cirurgia teve um papel de resgate de sua auto- 
estima, em especial no relacionamento com seu marido. Previamente à cirurgia, já tinha um diagnóstico de hipotireoidismo e interrompeu o tratamento medicamentoso para a patologia antes mesmo de submeter-se à cirurgia, porque "achava que tomava comprimidos demais". Ignorava - ou parecia ignorar a importância que teria para a diminuição de seu peso o correto tratamento da doença endocrinológica. Conta que, hoje em dia, sua grande dificuldade com a alimentação está no fato de que não consegue engolir carne. Apenas mastiga e joga fora. Observa que, muito embora não possa mais comer fartamente como antes, desenvolveu o hábito de sentir prazer em cozinhar para os outros da família, fato que compensa a sua limitação de ingestão de alimentos. Atualmente, está apresentando mudanças de atitudes. Relata que se conscientizou de que não poderia mais ser submissa a tudo e a todos. Não permanece mais tão passiva em casa, enquanto o marido sai com amigos para divertirse. Optou por "ter sua própria vida", sair e divertir-se com amigas, e "não mais esperar pelos outros para ser feliz”.

A entrevistada 3, solteira, 39 anos, conta que começou a comer compulsivamente e a engordar a partir dos 9 anos de idade, quando sofreu abuso sexual. Buscou na comida um refúgio para sua ansiedade. Quando atingiu níveis de obesidade mórbida, começou a provocar vômito para eliminar o alimento ingerido, uma vez que viu em um programa de televisão "que as modelos faziam isso para emagrecer”. Apresentava o entendimento de que a cirurgia iria eliminar toda a sua timidez. Conta que, depois do procedimento, passou a apresentar um comportamento semelhante "ao de uma adolescente" (sic), pois começou sair à noite, envolverse impulsivamente com homens (sendo que nunca havia tido relações sexuais até então), beber exageradamente e dirigir embriagada. Aponta que supervalorizou a cirurgia, esperando que, a partir desse procedimento, conseguisse obter "mudanças” em características que, na verdade, são intrínsecas à sua personalidade e não à sua aparência. Sua grande angústia e motivo de "sua depressão" (sic), neste momento, é o fato de estar voltando a engordar. Está sendo novamente avaliada pela equipe médica para ser reoperada.

A entrevistada 4, casada, 41 anos, conta que cresceu em uma família que discriminava pessoas obesas, apesar de seus pais também o serem. Seu pai, conta, era figura repressora e crítica quanto ao seu peso, referindo que "ninguém iria gostar dela por ser gorda”. Relata que se submeteu à cirurgia a fim de tratar problemas de saúde advindos de sua obesidade. Logo após o procedimento, já necessitou iniciar tratamento psiquiátrico, por apresentar medo intenso de retorno ao seu peso anterior. Acredita que sua maior frustração com a cirurgia está no fato de necessitar fazer uso de laxantes diariamente para manter a atividade intestinal preservada. Diante disso, expõe que deixou de tomar alguns medicamentos que eram necessários para tratar doenças conseqüentes à obesidade, mas teve de começar a utilizar outros que até então não precisava, desfazendo-se, assim, o mito de que a cirurgia seria um evento libertador de qualquer tipo de medicação e de constrangimento em sua vida.

A entrevistada 5, casada, 43 anos, aponta o casamento frustrado como principal causa de sua obesidade. Esse casamento, segundo a entrevistada, aconteceu por pressão da família. A escolha do marido foi realizada pelo seu pai, levando-se em consideração as origens genéticas do mesmo. A cirurgia tinha o claro objetivo de ser um divisor de águas em sua vida. Queria livrar-se da obesidade com o intuito de tornar-se forte o bastante para realizar "mudanças" e, quiçá, livrar-se do marido. Apesar de melhorar sua auto-estima, o emagrecimento desnudou o fracasso em sua vida conjugal e sexual. Uma vez que a obesidade não era mais um empecilho para o seu desempenho sexual, começou a perceber que existia um distanciamento entre o casal e uma forte incompatibilidade afetiva, e isso, então, passou a ser motivo de tristeza. A ansiedade e a angústia com que convive atualmente provocam-lhe vômitos, os quais apresentam uma forte carga emocional associada: "Sinto uma dor muito forte mesmo... Com uma angústia junto, que não é só dor física!”. Nos dias de hoje, desenvolveu o hábito de fumar (escondido do restante da família). Também terminou por envolver-se com outra pessoa, com quem vem mantendo um relacionamento extraconjugal.

A entrevistada 6 buscou a cirurgia basicamente com o intuito de recuperação de sua saúde, em especial procurando alívio para uma dor articular no joelho, devido à sobrecarga de peso. Em seu caso, a cirurgia "abriu as portas para ser uma pessoa mais corajosa", visto que teve de assumir o procedimento sem o apoio familiar e, nesse sentido, passou a enfrentar situações adversas com serenidade. Conta, por exemplo, que, após enfrentar a cirurgia de redução do estômago, encorajouse para realizar uma cirurgia oftalmológica, que vinha protelando há anos por não sentir-se apta para fazê-la. Aponta, também, a melhora significativa no relacionamento conjugal, inclusive com ganhos em sua vida sexual. Sua grande frustração, e motivo de seus sintomas depressivos, segundo interpretação da própria entrevistada, está no fato de que não perdeu o peso necessário para aliviar as dores do joelho, não alcançando, assim, o seu objetivo principal.

\section{Discussão}

A descrição de que a gordura pode ser vista como sinal de proteção e isolamento e de que, se esta for 
abruptamente retirada do indivíduo, poderá trazer-lhe caos e pânico ${ }^{15}$ encontra um ponto de sustentação na análise da fala da entrevistada 3. Observa-se que a timidez da entrevistada estava intrinsecamente ligada à sua personalidade, e não exatamente à sua gordura. $\mathrm{O}$ processo de ansiedade instalou-se no momento em que percebeu que mudanças mais profundas (emocionais) precisariam ser efetivadas, e não apenas as superficiais, vistas por meio da estética e do emagrecimento.

Para a entrevistada 1, a cirurgia era vista como uma técnica efetiva e prática para o resgate de sua autoestima. O aumento de peso que vem enfrentando póscirurgia está sendo motivo de angústia e até mesmo de pensamentos suicidas. Somado a isso, evidencia-se uma personalidade frágil, com dificuldade em lidar com as frustrações.

Em certos casos, a agressividade passou a ser um sentimento referido pelos pacientes após a cirurgia ${ }^{5}$, como por exemplo nas entrevistadas 5 e 1 . Em ambos os casos, certo grau de hostilidade com o cônjuge pode ser percebida; na paciente 1 , por ter melhorado sua autoestima, e isso ser um fato confrontador para o marido, que até então era uma pessoa desregrada; e na paciente 5 , por sentir um aumento de sua "energia vital” após o emagrecimento, tornando-se capaz de confrontar as agressividades do marido.

A entrevistada 5 deposita na técnica cirúrgica de emagrecimento um "renascer", um "tornar-se outra pessoa", a mais evidente dependência de um fator externo como verdadeiro propulsor de mudanças íntimas. Mesmo já tendo sido operada há mais de 1 ano, ainda não conseguiu efetivar o afastamento do marido.

Por outro lado, estudos realizados com obesos mórbidos que se submeteram à cirurgia bariátrica relatam o abandono, por parte desses pacientes, do seguimento do tratamento psicoterápico, assim como a importância desse seguimento posterior ${ }^{13}$. Em nossa pesquisa, somente uma paciente seguia acompanhamento psicoterápico no momento das entrevistas.

Para a entrevistada 2, ficou evidente a impossibilidade de dissociar a sua estética do meio profissional, sendo a obesidade um empecilho para o seu sucesso.

No que diz respeito à entrevistada 6 , a sua situação atual demonstra que apresentou expectativas além daquelas passíveis de serem alcançadas concernentes às perdas ponderais.

Na literatura médica, encontram-se relatos de casos de suicídio após a realização da cirurgia bariátrica ${ }^{16}$. Todos os pacientes já sofriam de quadro depressivo prévio, assim como já haviam sido submetidos a tratamento psiquiátrico.

A psicologia reconhece os benefícios que o emagrecimento pode trazer ao paciente, porém vê com menos entusiasmo a questão do emagrecimento rápido e acentuado promovido pelas técnicas cirúrgicas ${ }^{17}$. A obesidade funciona muitas vezes como um falso self mental e corporal, que protege um verdadeiro self frágil e mal-estruturado, atribuindose a isso a enorme dificuldade de emagrecimento em alguns pacientes ${ }^{18}$.

O uso da obesidade como defesa para enfrentamento de situações de vida ou até mesmo como fuga destas pode ser um complicador e fator gerador de ansiedade ${ }^{15}$.

Por conseguinte, é possível de se ter uma adequada percepção da complexidade com a qual um paciente se depara no tratamento de sua obesidade mórbida, assim como o grau necessário de maturidade da personalidade de alguém que venha a submeter-se a tal tratamento.

\section{Referências}

1. Bassette F. Após redução do estômago, 64\% voltam a ser obesos. Folha de São Paulo, São Paulo, 17 de jul. 2005. Cotidiano, p. C7.

2. Zwaan M. A comparision of different methods of assessing the features of eating disorders in post-gastric bypass patients: a pilot study. European Eating Disorders Review. 2004;12(6):380-6.

3. Olsson SA, Rydén O, Danielsson A, Nilsson-Ehle P. Weight reduction after gastroplasty: The predictive value of surgical, metabolic and psychological variables. Int J Obes. 1984;8(3):24558.

4. Rand C, Kuldau J, Robbins L. Surgery for obesity and marriage quality. JAMA. 1982;247(10):1419-22.

5. Benedetti C. De obeso a magro: a trajetória psicológica. São Paulo: Vetor; 2003.

6. Macìas JAG, Leal FJV. Personality assessment in morbid obesity. German J Psychiatry. 2002;5(4):90-4.

7. Larsen F. Psychological function before and after gastric banding surgery for morbid obesity. Acta Psychiatric Scand Suppl. 1999;359:1-57.

8. Powers PS, Rosemurgy AS, Coovert DL, Boyd FR. Psychosocial sequelae of bariatric surgery: a pilot study. Psychosomatics. 1988;29(3):283-8.

9. Larsen F, Torgersen S. Personality changes after gastric banding surgery for morbid obesity: a prospective study. J Psychosom Res. 1989;33(3):323-34.

10. Wooley SC, Wooley OW, Dyrenforth S. The case against radical interventions. Am J Clin Nutr. 1980;33 Suppl 2:465-71.

11. Heller DCL, Kerbauy RR. Redução de peso: identificação de variáveis e elaboração de procedimento com uma população de baixa escolaridade. Rev Bras Ter Comp Cog. 2000;2(1):31-52.

12. Cormillot AF. Obesidade. Buenos Aires: Médica Panamericana; 1977.

13. Franques A. Contribuições da psicologia na cirurgia da obesidade. São Paulo: Vetor; 2006.

14. Chimicati E. Obesidade: uma doença do afeto. $2^{a}$ ed. Belo Horizonte: Health; 2005.

15. Kahtalian A. Obesidade: um desafio. In: Mello Filho J, org Psicossomática hoje. Porto Alegre: Artmed; 1992. p. 273-8.

16. Omalu BI, Cho P, Shakir AM, Agumadu UH, Rozin L, Kuller LH et al. Suicides following bariatric surgery for the treatment of obesity. Surg Obes Relat Dis. 2005;1(4):447-9.

17. Békei M. Transtornos psicossomáticos en lá niñez y la adolescência. Buenos Aires: Nueva Vision; 1984.

18. Mello Filho JA. Winnicot: psicanálise em transicionalidade em Donald Winnicot estudos. Porto Alegre: Artmed; 1991. 